Heart Cardiac Sounds analysis using the Wigner distribution (WD) Method

\title{
S.M. DEBBAL
}

Genie -Biomedical Laboratory (GBM), Department of electronic Faculty of science engineering, university Aboubekr Belkaid , BP 119, Tlemcen 13000, Algeria

Corresponding Author: S.M. DEBBAL, Genie -Biomedical Laboratory (GBM), Department of electronic Faculty of science engineering, university Aboubekr Belkaid, BP 119, Tlemcen 13000, Algeria.

\section{Received Date: August 24, 2021; Accepted Date: September 07, 2021; Published Date: September 14, 2021}

Citation: S.M. DEBBAL. (2021) Heart Cardiac Sounds analysis using the Wigner distribution (WD) Method. J. Clinical Cardiology and Cardiovascular Interventions, 4(15); Doi:10.31579/2641-0419/216

Copyright: (c) 2021 S.M. DEBBAL, This is an open-access article distributed under the terms of the Creative Commons Attribution License, which permits unrestricted use, distribution, and reproduction in any medium, provided the original author and source are credited.

\begin{abstract}
This paper is concerned a "The Wigner distribution (WD)" analysis of the Heart cardiac (or phonocardiogram signals: PCG). The Wigner distribution (WD) and the corresponding WVD (Wigner Ville Distribution) have shown good performances in the analysis of non-stationary and quantitative measurements of the time-frequency PCG signal characteristics. It is shown that these transforms provides enough features of the PCG signals that will help clinics to obtain diagnosis.
\end{abstract}

Keywords: phonocardiogram; signal processing; sounds; heart cardiac's sounds; time frequency analysis; wigner distribution

\section{Introduction}

Heartbeat sound analysis by auscultation is still insufficient to diagnose some heart diseases. It does not enable the analyst to obtain both qualitative and quantitative characteristics of the phonocardiogram signals [1], [2]. Abnormal heartbeat sounds may contain, in addition to the first and second sounds, S1 and S2, murmurs and aberrations caused by different pathological conditions of the cardiovascular system [2]. Moreover, in studying the physical characteristics of heart sounds and human hearing, it is seen that the human ear is poorly suited for cardiac auscultation [3]. Therefore, clinic capabilities to diagnose heart sounds are limited.

The sound emitted by a human heart during a single cardiac cycle consist of two dominant events, known as the first heart sound S1 and the second heart sound S2 (Figure1), S1 relates to the closing of the mitral and tricuspid valves whilst S2 is generated by the halting of the aortic and pulmonary valves leaflets [1]. S1 corresponds in timing to the QRS complex in ECG (Electrocardiogram) and S2 follows the systolic pause in the normal cardiac cycle. Heartbeat sound analysis by auscultation only is still insufficient to diagnose some heart diseases. It does not enable the analyst to obtain both qualitative and quantitative characteristics of S1 and S2 of the phonocardiogram [1-2]. Moreover, in studying the physical characteristics of heart sounds and human hearing, it is seen that the human ear is poorly suited for cardiac auscultation [3]. Therefore, clinic capabilities to diagnose heart sounds are limited. In this paper we are interested in the study of the physical characteristics of the second heart sound $\mathrm{S} 2$ which consist of two major components in the spectrum of the signal. One of these components A2 is due to the closure of the aortic valve and the other $\mathrm{P} 2$ is due to the closure of the pulmonary valve. 


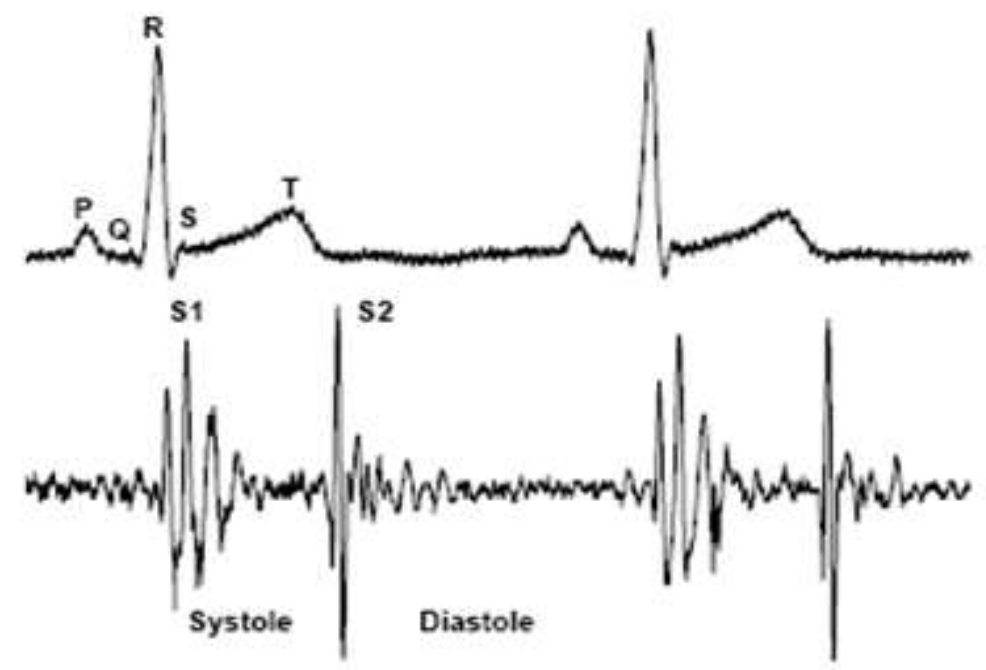

Figure 1: Correlation between the phonocardiogram signal (PCG and the electrocardiogram signal (ECG)

The aortic component is loudest than the pulmonic component. It is discernible at all the auscultation sites.It is best heard at the right base, with the diaphragm of the chest piece firmly pressed, whereas the pulmonic on a may only be audible at the left base, with the diaphragm of the chest piece firmly pressed.

The aortic component has higher frequency contents and generally precedes the pulmonary component because in normal heart activity the aortic valve closes before the pulmonary valve. The difference of time occurrence between these valves activities is known in medical community [4 - 6] as split. However in many diseases [1] this order of time occurrence may be reverse and its delay varies.

The characteristics of the PCG signal and other features such as heart sounds S1 and S2 location; the number of components for each sound; their frequency content; their time interval; all can be measured more accurately by digital signal processing techniques.

The Wigner distribution (WD) and the corresponding WVD (Wigner Ville Distribution) have shown good performances in the analysis of nonstationary signals. This comes from the ability of the WD to separate signals in both time and frequency directions. One advantage of the WD over the STFT is that it does not suffer from the time-frequency trade-off problem. On the other hand, the WD has a disadvantage since it shows cross-terms in its response. These cross-terms are due to the nonlinear behaviour of the WD, and bear no physical meaning. One way to remove these cross-terms is by smoothing the time-frequency plane, but this will be at the expense of decreased resolution in both time and frequency [7]. The WD was applied to heart sound signal it shows no success in displaying or separating the signal components in both the time and frequency direction [6], although it provides high time-and frequencyresolution in simple monocomponent signal analysis [8]

\section{Theoretical background of the Wigner Distribution (WD)}

The WD of two signals, $x(t), y(t)$, is defined via,

$W(t, \omega)=\int_{-\infty}^{+\infty} x\left(t+\frac{\tau}{2}\right) y^{*}\left(t-\frac{\tau}{2}\right) e^{-j \omega \tau} d \tau$
The auto WD is obtained when $x(t)=y(t)=\mathrm{s}(\mathrm{t})$, it is a bilinear function of the signal $\mathrm{s}(t)$. The WD, and can also be expressed as

$$
S(t, \omega)=\int_{-\infty}^{+\infty} s\left(t+\frac{\tau}{2}\right) s *\left(t-\frac{\tau}{2}\right) e^{-j \omega \tau} d \tau
$$

Where $\mathrm{t}$ and $\omega$ are respectively, the time and frequency variables, and * denotes the complex conjugate.

The WD had shown good applications in the analysis of non-stationary signal [9-11]. This comes from the ability of this method to separate signals in both time and frequency directions. The WD has a disadvantage that it is limited by the appearance of cross-terms. These cross-terms are due to the nonlinear behaviour of the WD, and bear no physical meaning. One way to remove these cross-terms is by smoothing the time-frequency plane [7], but this will be at the expense of decreased resolution in both time and frequency.

\section{Results and discussion of the Wigner distribution application}

Figure2 shows the WD application of the cardiac sound of the normal case (2a), the coarctation of the aorta (2b) and the innocent murmur (2c). One can notice here that the two principal components (A2 and P2) start to appear in the presence cross-terms. The WD results may be improved by increasing the sampling rate of original signal,, but it still suffers from the cross-terms problem because of the nonlinearity of the WD.

However the Wigner distribution (WD) has shown good performances in the analysis of non-stationary signals. This comes from the ability of the WD to separate signals in both time and frequency directions. One advantage of the WD over the STFT is that it does not suffer from the time-frequency trade-off problem. On the other hand, the WD has a disadvantage since it shows cross-terms in its response. These crossterms are due to the nonlinear behaviour of the WD, and bear no physical meaning. One way to remove these cross-terms is by smoothing the timefrequency plane, but this will be at the expense of decreased resolution in both time and frequency 

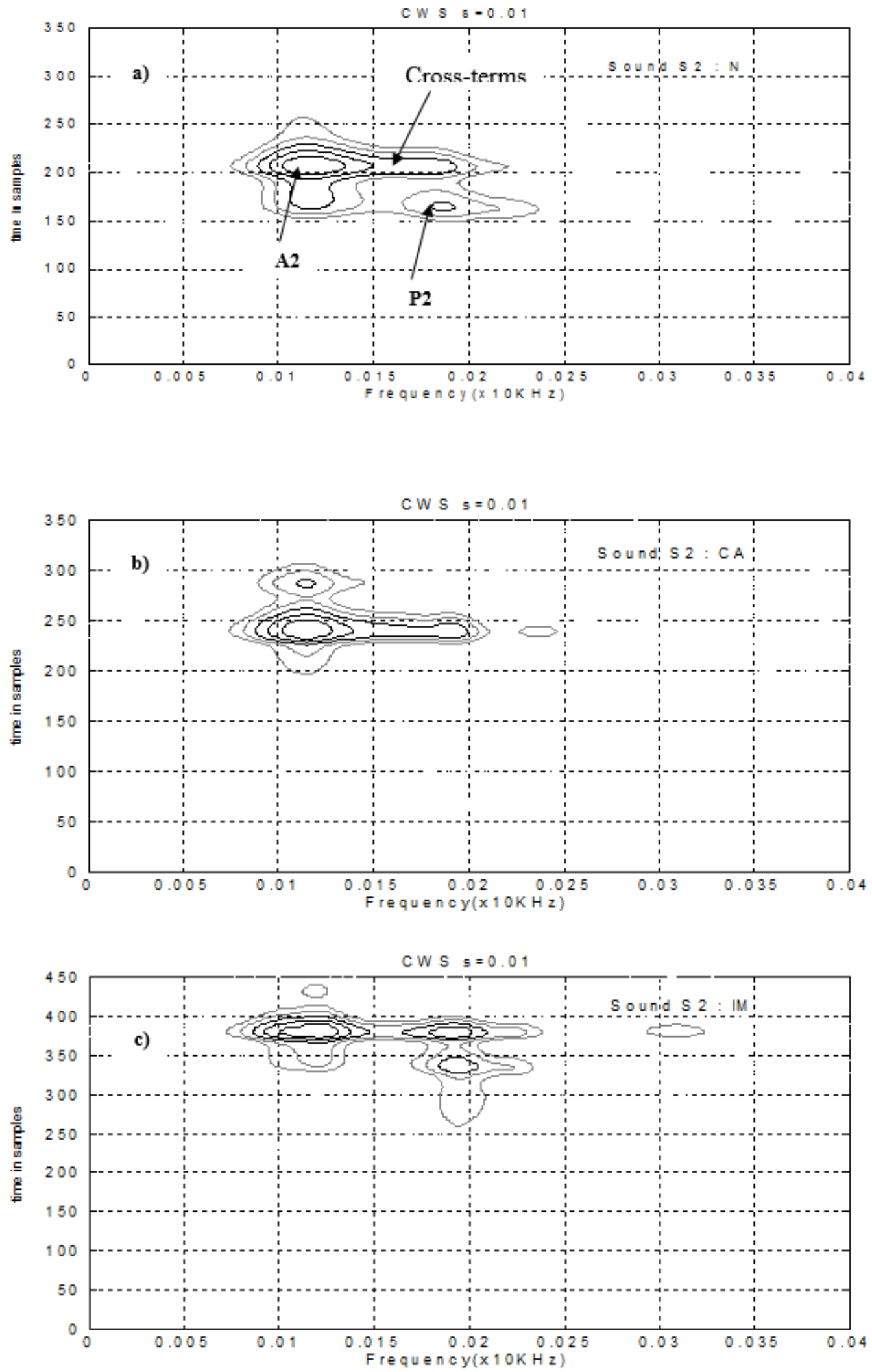

Figure2: The wigner distribution of the second cardiac sound S2 : a) the normal case , b) the coarctation of the aorta case (CA), c) the innocent murmur case (IM) 


\section{Conclusion}

The cardiac (heartbeat sound) cycle of phonocardiogram (PCG) is characterized by transients and fast changes in frequency as time progresses. It was shown that basic frequency content of PCG signal can be easily provided using FFT technique. However, time duration and transient variation cannot be resolved; the Wigner Distribution (WD) is a suitable technique to analyze such a signal. It was also shown that the graphic representation that provides a quantitative analysis simultaneously in time and frequency. It is therefore very helpful in extracting clinically useful information.

The measurement of the time difference between the A2 and P2 components in the sound $\mathrm{S} 2$, the number of major components of the sounds S1 and S2 and the frequency range and duration for all these components and sounds can be accurately achieved for Wigner Distribution (WD) simultaneously as was clearly illustrated.

It is shown that the WD Technic provides more information of the PCG signals with murmurs that will help physicians to obtain qualitative and quantitative measurements of the time and the time-frequency PCG signal characteristics and consequently aid to diagnosis.

\section{References}

1. RANGAYYAN,R.M and LEHNER ,R.J (1988). A review.CRC Critical Reviews in Biomedical Engineering 15 (3), 211-236.

2. RANGAYAN,R.M and LEHNER ,R.J (1988). Phonocardiogram signal analysis : a review.CRC Critical Reviews in Biomedical Engineering 15 (3), 211-236.
3. FEIGEN, L.P (1971). Physical characteristics of sound and hearing. American journal of Cardiology, 28 (2), 130-133.

4. TUTEUR, F.B (1988). Wavelet Transforms in signal detection. IEEE ICASSP, CH2561-9, 1435-1438.

5. GROSSMANN.A; HOLSCHNEIDER KRONLANDMARTINET.R and MORLET,J (1987). Detection of abrupt Changes in sound signal with the help of the wavelet transform. In : Inverse problemes : An interdisciplinary study. Advances in Electronics and Electron physics. Supplement 19 [New York Academic], 298-306.

6. OBAIDAT.M.S. Phonocardiogram signal analysis: techniques and performance comparison. Journal of Medical Engineering $\&$ technologie, vol 17, No 6 (November-December 1993), 221227.

7. BOASHAS, B (1993). Time-frequency signal analysis. In advances in spectrum Estimation, edited by S.Haykin, (NJ:Prentice-Hall).

8. WILLIAM J. WILLIAMS (1997). Time-frequency and wavelets in Biomedical Signal Processing. Edited by METIN AKAY. IEEE Press Serie in BME. 3-8.

9. Y.MEYER (1990). Ondelettes et Opérateurs. Tome1. Paris : Hermann.

10. S.MALLAT (1989). A theory for multiresolution signals decomposition: The wavelet representation. IEEE Trans.Pattern Anal.Machine Intell, vol 11, pp 674-693.

11. P.I.J. Keeton, F.S. Schlindwein, Application of Wavelets in Doppler Ultrasound, MCB University Press, Emerald Sensor Review, Vol. 17(1) 1997, pp. 38-45.

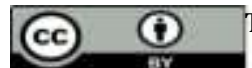

This work is licensed under Creative Commons Attribution 4.0 License

To Submit Your Article Click Here: Submit Manuscript

DOI: $10.31579 / 2641-0419 / 216$
Ready to submit your research? Choose Auctores and benefit from:

* fast, convenient online submission

* rigorous peer review by experienced research in your field

* rapid publication on acceptance

* authors retain copyrights

* unique DOI for all articles

* immediate, unrestricted online access

At Auctores, research is always in progress.

Learn more www.auctoresonline.org/journals/clinical-cardiology-andcardiovascular-interventions 